\title{
Evaluación del proceso de viverización para obtención de plantas de queñoa (Polylepis rugulosa Bitter) con distintos tipos de sustratos en la localidad de Chapiquiña, XV región de Arica y Parinacota, Chile
}

\author{
Nursery process evaluation forobtain queñoa plants \\ (Polylepis rugulosa Bitter) with different substrates in Chapiquiña, \\ Arica and Parinacota Region, Chile \\ Juan Francisco Trevizan Rispoli ${ }^{1}$, Ruth Angélica Aguilar Aliaga ${ }^{1}$
}

\begin{abstract}
RESUMEN
En un vivero ubicado en Chapiquiña, en la XV región de Arica y Parinacota, se evaluaron distintas mezclas de sustrato para la obtención de plantas de Polylepis rugulosa Bitter. Se realizaron diseños estadísticos completamente aleatorios, con prueba de significación de Tukey al 5\%. El sustrato óptimo para la obtención de plantas estuvo compuesto por tierra del sector de Chapiquiña, compost, turba y arena en proporción de 1:1:1:0,03. Se generaron plantas vigorosas con una altura acumulada durante el proceso de viverización de 10,07 cm, un diámetro a la altura de cuello de 1,78 mm y 19,8 hojas por planta, en promedio.
\end{abstract}

Palabras clave: Polylepis rugulosa, viverización, sustratos

ABSTRACT

In a breeding ground located in the Chapiquina Hydroelectric Power Station, Arica and Parinacota Region, different mixtures of substrates were evaluated to obtain plants of Polylepis rugulosa Bitter. The experiment was conduced using a randomized complete block design with four treatments and seven replications. A Tukey test was used to separate the means. The optimal substrate for obtain plants is made up with land of Chapiquiña, compost, crowd and sand in proportion of 1:1:1:0.03. Vigorous plants were generated, the accumulated height during the nursery process was $10.07 \mathrm{~cm}$; stem diameter was $1.78 \mathrm{~mm}$ and were obtain19.8 leaves per plant, on average.

Key words: Polylepis rugulosa, nursery, substrates, fertilization.

\section{Introducción y revisión bibliográfica}

Debido a la compleja y extensa geografía de Chile continental, existe una gran diversidad de ambientes con presencia de árboles y distintos tipos de formaciones vegetacionales a lo largo del territorio (García y Ormazábal, 2008).

En la zona cordillerana del extremo norte de Chile, se encuentran comunidades dominadas por dos árboles emparentados: los queñoales de la precordillera (Polylepis rugulosa Bitter), ubicados en la provincia de Parinacota y que se desarrollan entre los 3.400 - 4.100 msnm; y los queñoales de altura representados por Polylepis tarapacana
Phil, localizados en la meseta altiplánica (García y Ormazábal, 2008; González y Molina, 2015 y 2017) y capaces de habitar en forma natural a la mayor altitud del mundo, sobre los $4.500 \mathrm{msnm}$ (Cardozo y Miranda, 2006).

Desde el año 1985, cuando se realizó el simposio "Flora nativa arbórea y arbustiva de Chile amenazada de extinción", las queñoas de altura están catalogadas como especies vulnerables, y en el caso de las queñoas de precordillera se encuentran en peligro de extinción (Benoit, 1989; González y Molina, 2015 y 2017). Ambas especies han sido utilizadas durante mucho tiempo como leña, material de construcción y artesanía

\footnotetext{
$1 \quad$ Universidad de Tarapacá, Facultad de Ciencias Agronómicas. Arica, Chile.

* Autor por correspondencia: jftrevizan@gmail.com; jtrevizan@uta.cl
} 
principalmente, llegando al grado de exponerlas al estado de conservación en el cual se encuentran actualmente (Ríos, 1998).

Con el fin de generar información para realizar prácticas de reforestación de la queñoa de precordillera (Polylepis rugulosa Bitter), se buscó establecer y sistematizar el proceso de viverización de dicha especie y así optimizar la producción de plantas en la etapa de vivero con fines de conservación y reforestación. En la presente publicación se muestra la evaluación de distintas mezclas de sustratos para la obtención de queñoa de precordillera.

\section{Caracterización y descripción de Polylepis rugulosa Bitter (queñoa de precordillera). Clasificación taxonómica}

La planta en estudio se clasifica en Reino Plantae, División Magnoliophyla, Clase Magnoliopsida, Orden Rosales, Familia Rosaceae, Género Polylepis, Especie Rugulosa Bitter. Su nombre vulgar es queñoa de precordillera.

Polylepis es un género de árboles característicos de la cordillera de los Andes. Agrupa alrededor de 30 especies repartidas desde Venezuela hasta Argentina central, y los bosques que conforman se consideran entre los ecosistemas boscosos más amenazados del planeta (García y Ormazábal, 2008).

Polylepis rugulosa Bitter corresponde a un árbol perennifolio. Con ramas largas y dobladas, entrecruzándose tallos y ramas; posee una forma influenciada por las duras condiciones ambientales en las que crece (Núñez y Contreras, 1992). Mide entre 3-7 m de alto, corteza de color marrón rojizo que se desprende en grandes trozos y puede alcanzar hasta $1 \mathrm{~m}$ de diámetro. Sus hojas son imparipinnadas con 1-3 foliolos, ovalados, obovados a circulares, de 1,8-3,5 cm de ancho y 1,9-6,8 $\mathrm{cm}$ de largo, brillantes en el haz, con pelos blanquecinos en el envés; raquis lanoso (Kessler, 1995; González y Molina, 2015 y 2017).

Las inflorescencias colgantes miden de 4,5 a $10 \mathrm{~cm}$ de largo y están formadas por flores hermafroditas, actinomorfas, sin pétalos y poco vistosas. Las flores y frutos secos no se ven fácilmente ya que se hallan entre el follaje. El fruto es un aquenio lanoso, con 2-5 proyecciones planas de forma irregular con varias puntas (Muñoz y Serra, 2006).

Tanto la floración como la fructificación de Polylepis rugulosa son fenofases de larga duración en el tiempo (Belmonte et al., 1989). Estas fases y su duración están estrechamente relacionadas con la disponibilidad de agua, y pueden durar hasta 8 meses en zonas con mayor humedad y 4 meses en zonas más secas. La fructificación se puede extender hasta 8 meses, y se recomienda la recolección de frutos entre primavera y otoño, en los meses de octubre y mayo (Núñez y Contreras, 1992).

Los bosques de Polylepis presentan una regeneración natural basada en mecanismos sexuales y asexuales, es decir, regeneración proveniente de semillas y propagación vegetativa del tipo de acodos naturales, producidos generalmente por ramas laterales, que sin desprenderse del árbol, se ponen en contacto con el suelo y echan raíces (Núñez y Contreras, 1992).

\section{Principales amenazas y estado de conservación}

De acuerdo con García y Ormazábal (2008), existen estimaciones según las cuales, cerca del 45\% de la superficie original de bosques en Chile continental se ha perdido desde el siglo XVI, mientras que un $76 \%$ de los bosques remanentes se encontrarían en serio peligro de desaparecer. Entre los factores que más han incidido en la pérdida de superficies boscosas y árboles nativos están la extracción para leña, carbón y madera, los incendios forestales y, sobre todo, el despeje de terrenos para agricultura, ganadería e instalación de viviendas o poblados.

La primera instancia importante para la evaluación del estado de conservación de los árboles chilenos fue el simposio de expertos "Flora nativa arbórea y arbustiva de Chile amenazada de extinción", organizado por la Corporación Nacional Forestal (CONAF) en 1985, y que dio origen al Libro Rojo de la Flora Terrestre de Chile, publicado en 1989. Aquí, la queñoa de precordillera fue catalogada como especie vulnerable por CONAF, aunque según Benoit (1989), quedó clasificada en esta categoría bajo el nombre de Polylepis besseri.

Los bosques sudamericanos de Polylepis están considerados como los ecosistemas boscosos más amenazados (Renison y Cingolani, 1998). Han sido fuertemente empleados como combustible y en ciertos casos para construcción (Ríos, 1998).

Polylepis rugulosa es una planta poco frecuente, aunque no escasa, pues se encuentra con relativa factibilidad en los sectores donde de preferencia crece (Núñez y Contreras, 1992). El hecho de que 
se desarrolle en extensos territorios donde no existe otra planta en forma arbórea, la convirtió en la fuente de energía ideal para las actividades humanas. Esta explotación irracional fue especialmente intensa durante la construcción de las grandes obras públicas altiplánicas, además del uso desmedido por las actividades mineras (Serra et al., 1986; Kwoll, 1993). Como consecuencia de lo anterior, las poblaciones de $P$. rugulosa se han visto seriamente reducidas y actualmente se encuentran bajo fuerte presión, entre otros factores, por sus pocas posibilidades de incrementar la superficie poblacional a través de la regeneración natural, debido a la compleja dinámica que se genera dentro de los bosquetes (Núñez y Contreras, 1992). No obstante, las poblaciones mejor conservadas de la especie en Chile se hallan entre las localidades de Chapiquiña y Belén, pero está protegida solo en el Parque Nacional Lauca (García y Ormazábal, 2008). De acuerdo con la clasificación elaborada por la Unión Internacional para la conservación de la naturaleza IUCN, se clasifica como una especie Amenazada EN (en peligro), según González y Molina, 2015 y 2017).

\section{Tipos de reproducción para la obtención de nuevas plantas de queñoa}

De acuerdo con Orchard et al. (2009), los antecedentes acerca de los métodos de propagación del género Polylepis son escasos, al igual que las experiencias, y eso genera que las publicaciones de nuevos hallazgos de los métodos de propagación sean insuficientes.

Este género se caracteriza por presentar problemas reproductivos, por lo que encontrar la técnica apropiada en una localidad puntual es fundamental para la preservación de la especie (Vega et al., 2007).

\section{Materiales y métodos}

El ensayo tuvo lugar en la localidad de Chapiquiña, en el predio de la Central Hidroeléctrica
Chapiquiña, y se realizó desde enero hasta febrero del año siguiente.

La localidad de Chapiquiña está ubicada en la comuna de Putre, provincia de Parinacota, que forma parte de la región de Arica y Parinacota, al norte de Chile. Está situada a 120 kilómetros al oriente de Arica, sobre los $3.300 \mathrm{msnm}$.

De acuerdo con Pinto y Kirberg (2009), Chapiquiña posee un clima tropical xérico, el cual se caracteriza por presentar precipitaciones elevadas y la estación seca es mucho más larga que la húmeda. El promedio anual de precipitaciones en los últimos 30 años en la estepa arbustiva prealtiplánica ha sido de $141 \mathrm{~mm}$ para Chapiquiña (3.350 msnm).

\section{Diseño experimental y análisis estadístico}

En el ensayo para la evaluación de distintas mezclas de sustrato en la obtención de plantas de queñoa, se realizó un diseño completamente aleatorio simple, conformado por 3 tratamientos más un testigo (Tabla 1), con 7 repeticiones en cada tratamiento y 21 unidades experimentales. Cada unidad experimental estaba compuesta por 7 bolsas con sus plantas ya repicadas en ellas, lo cual genera una población total de 196 plantas de queñoa, dispuestas en una platabanda de 1,2 x $5 \mathrm{~m}$.

Se utilizó un análisis de varianza a una probabilidad $\mathrm{F}$ de 0,05 para establecer si existen diferencias significativas entre los tratamientos y la prueba de significación de Tukey nivel $\alpha 0,05$. Además para evaluar vigorosidad en las plantas se empleó la prueba de Friedman $\mathrm{X}^{2}$ al 0,05 con el fin de establecer diferencias entre los tratamientos.

\section{Llenado de bolsas con sustrato y repique}

Una vez listas las mezclas se llenaron los contenedores (bolsas de polietileno) y se realizó el repique, con plantas obtenidas de almácigo realizado en la misma localidad y utilizando solo plántulas con las primeras hojas verdaderas

Tabla 1. Tratamientos y detalle del ensayo de sustratos en queñoa.

\begin{tabular}{cl}
\hline Tratamiento de Sustratos & \multicolumn{1}{c}{ Composición de las mezclas de sustratos } \\
\hline T0 & 33\% Tierra del Sector $+33 \%$ Compost $+33 \%$ Turba $+1 \%$ Arena \\
T1 & 50\% Tierra del Sector $+50 \%$ Hojarasca de Queñoa \\
T2 & 50\% Tierra del Sector $+50 \%$ Compost \\
T3 & 50\% Tierra del Sector $+50 \%$ Turba \\
\hline
\end{tabular}


desarrolladas. Tras este repique, se procedió a la ubicación de acuerdo al diseño experimental en platabandas de $1,2 \times 5 \mathrm{~m}$, donde cupieron 280 plantas. Por lo tanto, se dispuso de una platabanda para el ensayo de sustratos y una platabanda para el ensayo de fertilización. A ambos ensayos se les aplicó Captan WP 80, un fungicida para prevenir cualquier ataque de hongos en la etapa inicial.

Es importante mencionar que el sustrato usado en el ensayo de fertilización fue tierra del sector de Chapiquiña (previamente harneada), compost, turba y una mínima parte de arena, en una proporción de 1:1:1:0,03.

\section{Observaciones y toma de datos}

Se realizaron monitoreos de las plantas cada 15 días y cada 30 días se efectuaron las mediciones de las siguientes variables:

- Altura de planta: utilizando una regla graduada en centímetros, se midió a ras del sustrato hasta la hoja más alta que se encontraba totalmente desarrollada. Este parámetro se relaciona con la capacidad fotosintética y la superficie de transpiración de las plantas.

- Diámetro de cuello: se mide con un pie de metro graduado en milímetros, en la base del tallo, con mucho cuidado para evitar daños a las plantas. Este valor es un indicador de la capacidad de transporte de agua hacia la parte aérea, resistencia mecánica y la capacidad de tolerar altas temperaturas.

- Índice de esbeltez: se obtiene al dividir los valores de altura de la planta y el diámetro de cuello. Este valor relaciona la resistencia de la planta con la capacidad fotosintética.

- Número de hojas: se consideraron hojas verdaderas plenamente expandidas como índice de desarrollo.

- Vigor de las plantas: se estimó visualmente utilizando como parámetro la escala de vigor del Centro Internacional de la Papa. Tabla 2 (CIP, 1996). Luego, a los datos obtenidos se les aplicó la prueba de Friedman para establecer diferencias estadísticas significativas.

\section{Resultados}

A continuación se presentan los análisis del efecto de diferentes mezclas de sustratos y su incidencia en el desarrollo de Polylepis rugulosa.
Tabla 2. Escalas de vigor del Centro Internacional de la Papa adaptadas y utilizadas para clasificar a las plantas de queñoa.

\begin{tabular}{ll}
\hline & Escala de Vigor del follaje \\
\hline 1 & Sin Vigor \\
3 & Poco Vigor \\
5 & Medianamente Vigoroso \\
7 & Vigoroso \\
9 & Muy Vigoroso \\
\hline
\end{tabular}

En la Figura 1 se puede apreciar la evolución a través del tiempo, en altura, de los tratamientos durante el periodo 2011. El mayor incremento se produjo siempre en T0, y llegó a alcanzar un promedio de $12,8 \mathrm{~cm}$ en el mes de diciembre, duplicando la altura promedio del resto de los tratamientos. Los demás tratamientos obtuvieron un crecimiento similar, y solo durante los últimos 4 meses se observa algún grado de diferenciación entre ellos. De acuerdo a la última fecha registrada, T1 alcanzó $6,4 \mathrm{~cm}$, seguido por T3 y T2 que solo lograron 4,8 y $3,6 \mathrm{~cm}$ de altura, respectivamente. Todos los tratamientos comenzaron con una altura similar: T0 con 2,7 cm; T1 con 2,1 cm; T2 con $2 \mathrm{~cm}$ y T3 con $2,3 \mathrm{~cm}$.

Los datos presentados en la Figura 2 muestran que T0 posee un DAC mayor que los otros tratamientos llegando a alcanzar 2,5 mm, seguido por $\mathrm{T} 1$ con 1,6 $\mathrm{mm}$, luego $\mathrm{T} 2$ con $1 \mathrm{~mm}$ y por último $\mathrm{T} 3$ con 1,4 mm de DAC. Esto indica que, al igual que en la altura de planta, T0 fue superior al resto, y lo demostró a partir del cuarto mes registrado. Anterior a ese registro, los diámetros del cuello

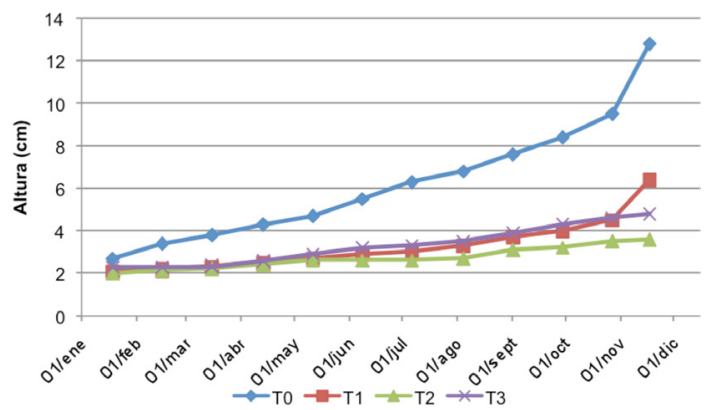

Figura 1. Altura de planta obtenida a lo largo del periodo de viverización. T0: tierra sector, compost, turba y arena en proporción 1:1:1:0,03. T1: tierra sector y hojarasca queñoa en proporción 1:1. T2: tierra sector y compost en proporción 1:1. T3: tierra sector y turba en proporción 1:1. 


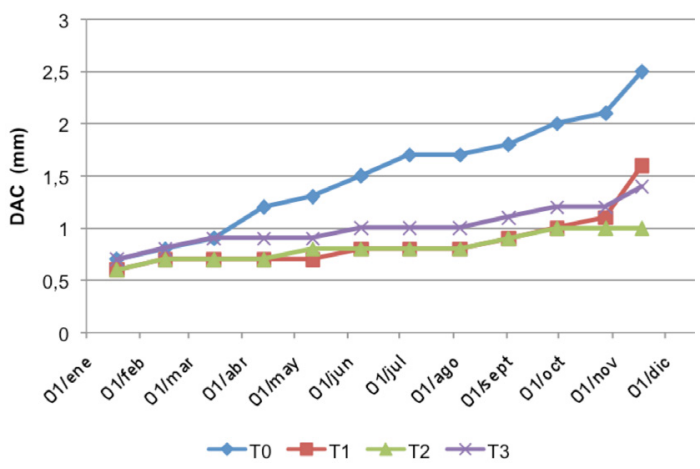

Figura 2. Diámetro a la altura del cuello (DAC), obtenido a lo largo del periodo de viverización. T0: tierra sector, compost, turba y arena en proporción 1:1:1:0,03.T1: tierra sector y hojarasca queñoa en proporción 1:1. T2: tierra sector y compost en proporción 1:1. T3: tierra sector y turba en proporción 1:1.

en los tratamientos fueron similares entre sí: T0 y T3 tenían $0,7 \mathrm{~mm}$ de grosor de cuello, mientras que $\mathrm{T} 1$ y $\mathrm{T} 20,6 \mathrm{~mm}$.

En la Figura 3 se observa claramente que el desarrollo foliar, basado en el conteo de hojas desarrolladas completamente expandidas, es mayor en T0 alcanzando en promedio, durante el último registro, 20 hojas por planta. Los tratamientos T1 y T3 obtuvieron en promedio 10 hojas, mientras que T2 logró un desarrollo de 7 hojas como promedio por planta. Todos los tratamientos poseen un promedio inicial de 3 hojas por planta, el cual fue aumentando gradualmente durante el periodo de viverización. A partir del segundo mes registrado, T0 muestra su superioridad en comparación con los demás tratamientos.

En la Figura 4 se observan las variables altura (cm), diámetro a la altura del cuello (DAC) (mm) y

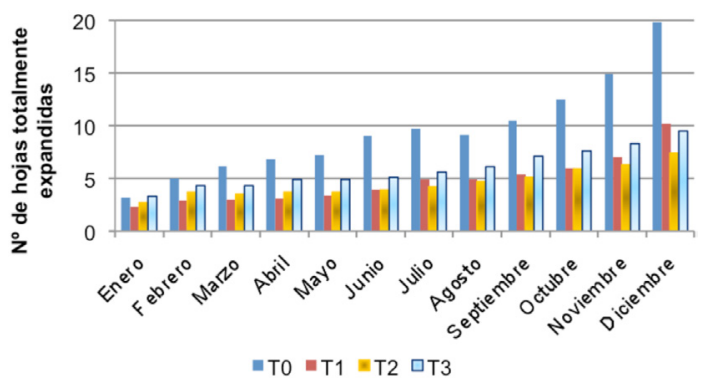

Figura 3. Número de hojas totalmente expandidas obtenidas a lo largo del periodo de viverización. T0: tierra sector, compost, turba y arena en proporción $1: 1: 1: 0,03 . T 1$ : tierra sector y hojarascaqueñoa en proporción 1:1. T2: tierra sector y compost en proporción 1:1. T3: tierra sector y turba en proporción 1:1.

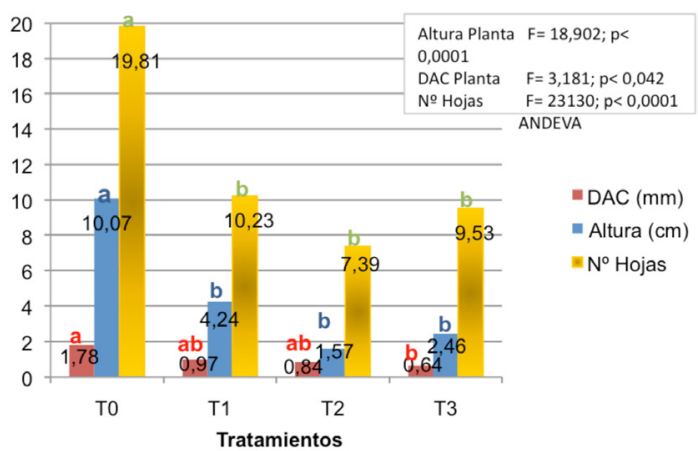

Figura 4. Comparación entre los tratamientos y las variables acumuladas dealtura $(\mathrm{cm})$, diámetro a la altura del cuello (DAC) (mm) y número de hojas totalmente expandidas. T0: tierra sector, compost, turba y arena en proporción 1:1:1:0,03. T1: tierra sector y hojarasca queñoa en proporción 1:1. T2: tierra sector y compost en proporción 1:1. T3: tierra sector y turba en proporción 1:1.(Valores con letras diferentes difieren estadísticamente, según la prueba de Tukey a p $\leq 0,05$ ).

número de hojas por planta, acumuladas durante el proceso de viverización. De acuerdo con el ANDEVA realizado, existen diferencias altamente significativas entre los tratamientos y las distintas variables evaluadas. T0 se muestra superior a los demás, obteniendo una altura acumulada de $10,07 \mathrm{~cm}$, un DAC acumulado de $1,78 \mathrm{~mm}$ y número acumulado de hojas de 19,81. Los tratamientos T1, T2 y T3 son estadísticamente similares entre sí, para las variables altura y número de hojas, siendo T2 el que obtuvo una menor acumulación de estas variables con un $1,57 \mathrm{~cm}$ de altura y 7,39 hojas. Mientras que T3 fue el tratamiento que menor DAC alcanzó con solo $0,64 \mathrm{~mm}$. El coeficiente de variación para altura y DAC fue de un $50,72 \%$ y $69,45 \%$, respectivamente, lo que indica que el análisis estadístico para dichas variables tuvo una baja confiabilidad. Mientras que el coeficiente de variación para el número de hojas fue de $15,52 \%$, lo cual es aceptable para las condiciones de campo del ensayo, ya que de acuerdo con la prueba de Tukey, el coeficiente de variación no debe ser mayor a un 30\%. Respecto al DAC, este es un factor escasamente variable entre los tratamientos aplicados, es decir, el sustrato tiene escasa influencia en este parámetro.

En la Figura 5 se muestran los datos tomados en el último mes de registro correspondiente a noviembre de 2011, y se aprecian diferencias significativas entre los tratamientos para cada variable en estudio. T0 y T1 fueron superiores al resto, en las variables ancho y largo de hoja, 


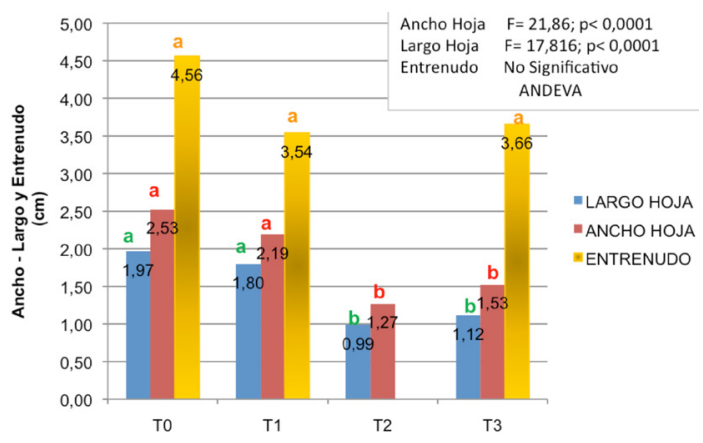

Figura 5. Comparación entre los tratamientos y las variables entrenudo, ancho y largo de hojas totalmente expandidas. T0: tierra sector, compost, turba y arena en proporción 1:1:1:0,03. T1: tierra sector y hojarasca queñoa en proporción 1:1. T2:tierra sector y compost en proporción 1:1. T3: tierra sector y turba en proporción 1:1.(Valores con letras diferentes difieren estadísticamente, según la prueba de Tukey a p $\leq 0,05$ ).

mientras que en entrenudo, todos los tratamientos son similares entre sí.

Para largo de hoja T0 obtuvo 2,53 cm, mientras que T1 alcanzó $2,2 \mathrm{~cm}$. Para la variable ancho de hoja T0 y T1 obtuvieron 1,96 y $1,8 \mathrm{~cm}$, siendo superiores a los T2 y T3. En T2 no fue posible lograr largo de entrenudo debido a la baja altura que presentaban las plantas, y solo se observaban hojas desarrolladas y el ápice juntos formando una roseta. De acuerdo al análisis de varianza, el coeficiente de variación del largo de entrenudo fue de $28,9 \%$, lo que indica un grado de confiabilidad aceptable de los datos. Los coeficientes de variación para ancho y largo de hojas fueron de 18,36\% y 19,01\%, respectivamente, lo cual evidencia confiabilidad en los datos obtenidos (ver Anexo 3).

Se debe considerar que al ser provenientes de semillas, las plantas presentan diferencias entre sí en cuanto a su desarrollo, y esto genera una alta variabilidad de los datos obtenidos, lo cual también influye en el coeficiente de variabilidad del ANDEVA.

Según la Tabla 3, y de acuerdo al análisis de varianza y prueba de significación realizada, T0 presenta en promedio un IE igual a 5, lo cual indica su adecuada relación entre altura de planta y grosor de tallo, frente al resto de los tratamientos. Los tratamientos T1, T2 y T3 son similares entre sí, no hay diferencia estadística y solo alcanzan un índice de esbeltez bajo 5, lo que evidencia una baja altura de planta de acuerdo a sus respectivos DAC. El coeficiente de variación para este caso alcanzó a un $16,86 \%$.
Con respecto al análisis de vigor, se aplicó la estadística de Friedman del $\mathrm{X}^{2}(\mathrm{p}<0,05)$, y de acuerdo con los resultados obtenidos (ver Anexo 5), existen diferencias estadísticas entre los tratamientos, las cuales nos indican que T0 fue superior a los demás, siendo vigoroso. Por el contrario, vemos a $\mathrm{T} 1$ con mediana vigorosidad y $\mathrm{T} 2$ - T3 con poco vigor. La escala aplicada corresponde a la utilizada por el CIP (Centro Internacional de la Papa, 1996).

De acuerdo a los resultados presentados para el ensayo de distintas mezclas de sustratos en el proceso de viverización, se observa claramente que T0 (mezcla compuesta por 33\% tierra del sector $+33 \%$ compost $+33 \%$ turba $+1 \%$ arena) fue el mejor tratamiento y es estadísticamente superior al resto en todas las variables estudiadas. Lo siguen T1 $(50 \%$ tierra del sector $+50 \%$ hojarasca de queñoa), T3 (50\% tierra del sector $+50 \%$ turba) y T2 (50\% tierra del sector $+50 \%$ compost). No obstante, se debe tener en cuenta que entre estos últimos 3 tratamientos no existieron diferencias estadísticas.

Entre los factores que influyen en la obtención de plantas de vivero están la temperatura, agua y disponibilidad de nutrientes (Buchner, 2007). De acuerdo al análisis de sustrato y agua que se realizó al comenzar con el ensayo, los resultados arrojados por el análisis de suelo y fertilidad muestran, en general, que los sustratos utilizados poseen características poco favorables para el desarrollo de plantas, puesto que son moderadamente salinosbásicos. La disponibilidad de nitrógeno no pudo ser detectada y el fósforo disponible resultó inferior a 8 $\mathrm{mg} \mathrm{kg}-1$, indicando un valor bajo para el desarrollo óptimo de plantas, mientras que los valores de

Tabla 3. Comparación entre los tratamientos y sus respectivos índices de esbeltez y vigorosidad obtenida al final del periodo de viverización en el ensayo de sustrato. T0: tierra sector, compost, turba y arena en proporción 1:1:1:0,03.

T1: tierra sector y hojarasca queñoa en proporción 1:1. T2: tierra sector y compost en proporción 1:1. T3: tierra sector y turba en proporción 1:1(Valores con letras diferentes difieren estadísticamente, según la prueba de Tukey a p $\leq 0,05$ ).

\begin{tabular}{ccl}
\hline Tratamientos & $\begin{array}{c}\text { Índice de } \\
\text { Esbeltez (IE) }\end{array}$ & \multicolumn{1}{c}{ Vigor de plantas } \\
\hline T0 & $5(\mathrm{a})$ & Vigoroso $(7,3)$ \\
T1 & $3,9(\mathrm{~b})$ & Medianamente Vigoroso (5) \\
T2 & $3,6(\mathrm{~b})$ & Poco Vigor $(3)$ \\
T3 & $3,5(\mathrm{~b})$ & Poco Vigor $(3,6)$ \\
\hline
\end{tabular}


potasio son muy altos a excepción de T3, el cual presentó un valor bajo. Solo en el contenido de materia orgánica se observan diferencias claras, siendo T0 y T3 los que obtuvieron un valor aceptable para el adecuado desarrollo de plantas, según INIA (2012). Con respecto al agua utilizada en los riegos, presenta valores de $\mathrm{pH}$ ligeramente básico y una concentración de bicarbonato elevada al igual que el boro. Esto provocaría en las plantas algún grado de toxicidad y posiblemente clorosis férrica; sin embargo, la rusticidad de la queñoa la hace tolerante a estas condiciones. En términos generales, es un agua de buena calidad que se puede utilizar para los ensayos de sustrato y fertilización de queñoa.

Las precipitaciones y temperaturas registradas por la Central Hidroeléctrica Chapiquiña en enero y febrero evidencian precipitaciones en los meses de verano (diciembre-marzo), que corresponden a lluvias orográficas causadas por masas de aire provenientes de la región del Amazonas, debido a la estación lluviosa en esa zona. Dichas precipitaciones llegaron a alcanzar los 5,3 $\mathrm{mm}$ en el mes de enero de 2011, mientras que en abril la precipitación mínima fue de $0,22 \mathrm{~mm}$. Las temperaturas promedio anuales registradas fueron de $20,9^{\circ} \mathrm{C}$ como máxima, $5,8^{\circ} \mathrm{C}$ mínima y en promedio $13,4^{\circ} \mathrm{C}$. La oscilación térmica promedio anual fue de $15,1^{\circ} \mathrm{C}$.

La queñoa es una especie que ha evolucionado en zonas donde las condiciones edáficas y climáticas no son las más favorables para el desarrollo de vegetación, por lo cual estaría adaptada a suelos de baja calidad productiva como el que se encuentra en el sector de Chapiquiña, donde se realizó el estudio, y que corresponde a su hábitat natural (Orchard et al., 2009). Por ello las temperaturas y precipitaciones eran las adecuadas para su normal desarrollo, sin influir mayormente. Además, el endemismo juega un rol preponderante, ya que ha logrado adaptarse al sector precordillerano y por ende al tipo de clima.

La disponibilidad de agua y los nutrientes se relacionan estrechamente ya que están en función del tipo de sustrato en el cual se desarrollan las plantas (Fuentes, 1999; Peñuelas y Ocaña, 1996). De acuerdo con esto, la mezcla de sustrato utilizada en T0 (tierra del sector, compost, turba y arena) fue la mejor, logrando combinar cada una de las propiedades de cada elemento utilizado y generar un sustrato de cultivo satisfactorio para producir plantas de queñoa en un medio óptimo de adecuada retención de agua, aireación y disponibilidad de nutrientes. Posiblemente, también hay un efecto favorable por su elevada materia orgánica, cercana al 3,38\%, aun cuando el análisis de agua y suelo no muestren las mejores características de ambos, sino que más bien son adversas con un alto $\mathrm{pH}(8,04), \mathrm{Ce}(4,6 \mathrm{mS} / \mathrm{cm})$, lo que significa un sustrato salino básico (Fuentes, 1999). No obstante, este efecto no se refleja en el crecimiento ni en la vigorosidad de las queñoas. Esto concuerda con Barcelóet al. (2001), quien señala que las plantas jóvenes, al no presentar dificultades de agua y minerales, aumentan en biomasa, produciendo un incremento de células meristemáticas y del área fotosintética, lo cual potencia el crecimiento.

En cambio, las mezclas constituidas en proporciones de 1:1 como son los tratamientos T1 (tierra del sector y hojarasca de queñoa), T2 (tierra del sector y compost) y T3 (tierra del sector y turba), no parecen ser las mejores, puesto que cada componente (exceptuando la tierra del sector que es igual para todos) proporciona características diferentes que no logran complementarse entre sí. Así tenemos que al adicionarle hojarasca de queñoa a la tierra del sector, solo se proporciona un material con partículas de gran tamaño, con la mínima disponibilidad de nutrientes que se puede encontrar en la hojarasca bajo el dosel de árboles de queñoa, puesto que al ser lavado con los continuos riegos, la hojarasca de queñoa pierde nutrientes. Por ende, esta mezcla salina y moderadamente básica no proporciona los requerimientos adecuados para un normal desarrollo, aunque frente a T2 y T3, T1 es mejor según los datos estadísticos obtenidos.

Según la mezcla de T2, el compost adicionaría mayor cantidad de materia orgánica y por ende mejor disponibilidad de nutrientes (Fuentes, 1999), por lo cual los resultados esperados difieren de los obtenidos, puesto que el crecimiento en general de la planta fue el menor en comparación con los demás tratamientos. Entre las posibles causas se podría decir que los altos valores de conductividad eléctrica $(6,07)$ y $\mathrm{pH}(8,17)$ producen un medio de cultivo salino-básico, generando poca disponibilidad principalmente de fósforo $(\mathrm{P})$. Al parecer esto queda en evidencia en el T2, que obtuvo el menor ancho y largo de hojas, así como un entrenudo, que después de 11 meses no se apreciaba por la baja altura de las plantas. Esto concuerda con Fuentes (1999), quien indica que al intervenir el $\mathrm{P}$ en procesos de crecimiento y síntesis de los 
componentes de las plantas, su deficiencia ocasiona un desarrollo débil, tanto del sistema radical como de la parte aérea, y ello se traduce en queñoas de menor desarrollo y vigor.

Ahora bien, según la mezcla de T3, la turba adicionada proporciona un medio de cultivo mucho más suave para el crecimiento de las raíces, así como un sustrato con una mayor capacidad de retención de agua debido a su alto contenido de materia orgánica $(3,59 \%)$, en comparación con el resto de los tratamientos. Sin embargo, al ser un sustrato inerte, no proporciona una cantidad adecuada de elementos nutritivos, lo cual conlleva un factor limitante para el correcto crecimiento y desarrollo de las queñoas. Pese a que la conductividad eléctrica indica un sustrato ligeramente salino con un $\mathrm{pH}$ básico, el desarrollo de las plantas fue uno de los más deficientes y así lo demuestra el análisis estadístico.

Las queñoas se desarrollan en muchas zonas altoandinas donde existen condiciones de suelo desfavorables, debido a que las bajas temperaturas y la aridez limitan la descomposición de la materia orgánica y el reciclaje de nutrientes, sobre todo en la disponibilidad de nitrógeno y fósforo, que parecen ser los factores limitantes para el desarrollo de las plantas (Kessler, 2006).

Quizás es por esto que, aun cuando las plantas de queñoa estuvieron sometidas a diferentes mezclas de sustrato que, según el análisis de fertilidad presentaban valores muy por debajo de los rangos óptimos para obtener plantines, lograron desarrollarse en algunos sustratos de mejor manera que en otros $\mathrm{y}$ todos mostraron un incremento en las variables en estudio. De la misma forma, al observar el ensayo en terreno era posible notar la gran variabilidad en cuanto a altura de plantas que existía entre los tratamientos y dentro de una misma unidad experimental, lo que se tradujo en coeficientes de variación elevados (sobre el 50\%) para las variables altura de plantas, DAC y entrenudo. El género Polylepis, por su evolución y endemismo, muestra una gran tolerancia a condiciones ambientales extremas como temperaturas bajas y largos periodos secos, con una alta variabilidad genética (Kessler, 2006), favoreciendo su desarrollo en sectores como Chapiquiña.

\section{Conclusiones}

Para el ensayo de diferentes mezclas de sustrato y su incidencia en la obtención de plantines de Polylepis rugulosa, el sustrato que presentó mejor respuesta para el óptimo desarrollo de plantines fue el testigo compuesto por tierra del sector, compost, turba y arena en proporción 1:1:1:0,03. Se generaron plantas de mayor tamaño y calidad frente al resto de los tratamientos con tierra del sector y agregados como hojarasca de queñoa, compost y turba, que en general estaban compuestos en una proporción de 1:1.

Las hipótesis de trabajo iniciales fueron demostradas, y se evidenció que el suelo de Chapiquiña (tierra del sector), con diferentes agregados orgánicos, causa un efecto significativo en el crecimiento y desarrollo de los plantines de queñoa.

\section{Literatura Citada}

Barceló, J.; G. Nicolás y B. Sabater.

2001. Fisiología Vegetal. Ed. Pirámide. Madrid, España. $566 \mathrm{p}$.

Belmonte, E.; Bahamondes, R.; Morales, C.

1989. Fenología de Polylepis besseri en la precordillera andina de la I región (sector Chapiquiña). Nota Técnica. Departamento Técnico Corporación Nacional Forestal (CONAF), Arica, Chile. 34 p.

Benoit, I.

1989. Red Book on Chilean Terrestrial Flora. Part One. Corporación Nacional Forestal (CONAF). Santiago, Chile. 151 p.

Buchner, C.

2007. Respuesta inicial de una plantación de Nothofagusdombeyi (MIRB) OERST a distintas dosis de fertilizante, en la recordillera de la Costa de Valdivia. Universidad Austral de Chile. Facultad de Ciencias Forestales. Valdivia, Chile. 55 p.

Cardozo, C.; Miranda. E.
2006. Especie de altura que prospera en el norte de Chile: Conservación de los bosques de queñoas. Chile Forestal (Chile), 2006: 7-10.

Centro Internacional de la Papa. 1996. Informe Anual. CIP. Lima, Perú. 60 p.

Fuentes, Y.

1999. El suelo y los fertilizantes. Ed. Mundi-Prensa. Madrid, España. 483 p.

García, N.; Ormazábal, C.

2008. Árboles nativos de Chile. Enersis S.A. Santiago, Chile. $196 \mathrm{p}$.

González, J.; Molina, J.

2015. Flora presente en la región de Arica y Parinacota. Arica, Chile. 115 pp.

González J. S.; Molina, J.J.

2017. Flora nativa de la región de Arica y Parinacota. Ediciones Universidad de Tarapacá. Arica, Chile. 233 p. 
Instituto de Investigaciones Agropecuarias.

2012. Interpretación de resultados análisis de suelo. Disponible en: http://www.inia.cl/link.cgi/suelo/resultados/7706. Consultado: 20/jul/2012.

Kessler, M. 1995.

The genus Polylepis (Rosaceae) in Bolivia. Candollea, 50: 131-171.

Kessler, M.

2006. Bosques de Polylepis. En: Moraes, M.; Ollgaard, B.; Kvist, L. P.; Borchsenius, F.; Bals-lev, H. (eds.). Botánica Económica de los Andes Centrales Universidad Mayor de San Andrés. La Paz, Bolivia. pp. 110-120.

Muñoz, M.; Serra, M.

2006. Documento de Trabajo. Estado de conservación de las plantas de Chile. MNHN-CONAMA. Disponible en: : http://www.conama.cl/clasificacionespecies/Anexo_tercer_ proceso/Polylepis_rugulosa_besseri.doc Consultado: 5/ Ene/ 2011.

Núñez, E.; Contreras, J.

1992. Informe Final Primera Fase Estudio Técnicas de Producción y Planificación en queñoa (Polylepisbesseri). Corporación Nacional Forestal. Región de Tarapacá. Iquique, Chile. 52 p.
Orchard, C., Mersey, L.; Stern, S., Green, D.; Zepeda, O. 2009. Aproximaciones a la propagación de Polylepis tarapacana Phil. en el altiplano de la I región de Iquique. Ambiente Forestal. Chile, 4 (7): 35-42.

Peñuelas, J.; Ocaña, L. 1996. Cultivo de plantas forestales en Contenedor. MAPA/ Ediciones Mundi-Prensa. Madrid, España. 190 p.

Pinto, R.; Kirberg, A.

2009. Cactus del extremo norte de Chile. Ed. Autoedición. Chile, Santiago. 250 p.

Ríos, R.

1998. Estudios de la distribución y caracterización ecológica de las poblaciones locales de queñoa de altura (Polylepis tarapacana Phil.) en el sector de la provincia de Iquique, Tarapacá. Santiago, Chile. 61 p.

Serra, V.; Gajardo, M., Cabello, L.

1986. Ficha Técnica de especies amenazadas Polylepis besseri Hieron. "Queñoa". Universidad de Chile, Facultad de Ciencias Agrarias y Forestales. Dpto. de Silvicultura y Manejo. Santiago, Chile. 21 p.

Vega, C.; Bermejo, J.;Villegas, G.; Quezadal, J.; Aguilar, M.; Conde. E.

2007. Massive propagation of Polylepis tomentella Weddell ssp. nana through in vitro culture techniques. Ecología Boliviana, 42 (2): 102-120. 
